\title{
FTMP-based Kink Deformation and Strengthening Mechanisms for Mille-feuille Structures
}

\author{
Y Nawa'*, T Hasebe ${ }^{2}$ \\ 1. Graduate School of Kobe University, Japan \\ 2. Kobe University, Japan
}

\begin{abstract}
This study aims at reproducing deformation-induced kink morphologies, together with the recently-reported AE measurement-based energy releasing characteristics, based on FTMP (Field Theory of Multiscale Plasticity)-incorporated crystal plasticity finite element simulation for single crystal Mg under c-axis plane strain compression condition. Two models either with the well-defined deformation twin system or a rank-1 connectionbased kink system are considered, respectively, as the projection direction for the incompatibility tensor to be used in the constitutive model. The twin model yields "kink-like morphology" of growing kind, but fails to capture one of the energy releasing characteristics. The kink model, on the other hand, is demonstrated to be able to reproduce basically all the features, i.e., not only the "kink" morphology but also the AE energy-based features.
\end{abstract}

\section{INTRODUCTION}

Kink-Strengthening, observed in a group of $\mathrm{Mg}$ alloys associated with the mille-feuille structures, has attracted many attentions in recent years [1-5]. Kink deformation, initially observed within a long-period stacking ordered (LPSO) phase [1-5] in Mg-Zn-rare earth alloys after compression, is considered to be a common deformation mode to layered or fibrous materials. Figure 1 shows an example of the kink bands formed in Mg-based LPSO phase [5] under compression. Clarifying, for example, the critical conditions for the kinking in the presence of such "mille-feuille-like structures" in general, can lead us to propose a new guideline for material designs.

Aizawa et al. [6] have recently reported based on AE measurement that there exists "scale free"-like, rather peculiar, kinking-related energy releasing characteristics during c-axis compression of 18R-LPSO single phase DS (directionally-solidified) Mg alloy samples in parallel to the solidification direction, both before and after the macro-yielding. This finding implies the following two, rather specific features of the LPSO structure, i.e., (i) multiscale aspects of the kink formation dynamics, having "no specific" scale, especially for larger sized kinks, accompanied by "avalanche-like" processes, and (ii) the kinking processes are following "precursor" counterpart "before” the macro-yielding takes place. These two features are further corroborated by the similarities also found in in the return maps both of the wait time and the energy differences for the measured AE signals.

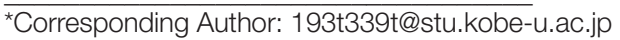




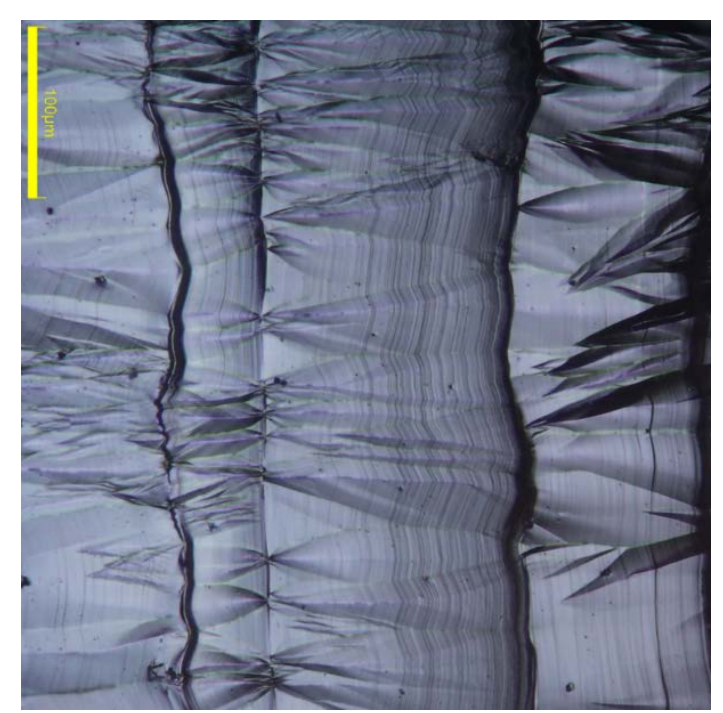

Figure 1: Kinked microstructure observed in 4\% compressed 6Zn-9Y DS Mg alloy (Courtesy of Prof. K. Hagihara).

There have been no established models proposed to date for the kinking, however, that are capable of describing and simulating both the nucleation and the subsequent growths of the kinking regions. The difficulties of dealing with such deformation-induced kinking come down to how to introduce the microscopic degrees of freedom responsible for the targeted phenomenon (mode) as an alternative to the other deformation modes in rational manners. Field theory of multiscale plasticity (FTMP) [7-22] is a comprehensive theoretical framework that enables direct and effective treatments of "inhomogeneities" in general, in terms not only of such differential geometric quantities, based on the non-Riemannian plasticity [23,24], but also of their evolutionary aspects of deformation-induced kinds. A series of FTMP-based approaches [7-22] have revealed so far that the "incompatibility" tensor is the one that possesses a dominant ability to help drive the evolutions of such "inhomogeneities".

This study attempts to reproduce basic and essential features of the targeted phenomena associated with the kink formation processes based on a series of preliminary analyses, making use of the already-constructed constitutive model [7-12] for the slip-based mode, accommodated with the FTMP-based incompatibility model for deformation twinning $[18,19]$ in two steps, against one of the simplest simulation setups with two symmetric twining planes under plane-strain c-axis tension mode. The first step utilizes the twin systems accommodated with newly-introduced orientation modification scheme, while the second step assumes the Rank-1 connectivity condition [20] in the place of the twin systems, without using the scheme. For both the steps, we examine a couple of the above-mentioned features about energyreleasing characteristics [6], focusing on the formations and further growths of "kink" morphologies. 


\section{THEORETICAL BACKGROUND}

2.1. Brief overview of FTMP

The authors have advocated a theoretical framework called FTMP (field theory of multiscale plasticity) [7-22], aiming at rationally dealing with the spatio-temporally deformation-induced evolving inhomogeneous fields in a comprehensive manner, by viewing them from three standpoints of (a)description, (b)evolution and (c)cooperation [7,14]. The theory mainly utilizes the differential geometric quantities, i.e., torsion tensor $S_{i j}^{k}$ (or $\boldsymbol{S}$ ) and curvature tensor $R_{l i j}^{k}$ (or $\boldsymbol{R}$ ), for (a) and (c), based on the non-Riemannian plasticity [23,24], advocated by K. Kondo and his colleagues. They, in the context of continuum mechanics of materials, correspond to the dislocation density tensor $\alpha_{i j}$ (or $\boldsymbol{\alpha}$ ) and the incompatibility tensor $\eta_{i j}$ (or $\boldsymbol{\eta})$, respectively, because the former counts the closure failure of a circuit encircling the defected field, whereas the latter represents the indeterminacy of displacement vector after its parallel displacement along the circuit. They are defined as curls of plastic distortion and plastic strain tensors, i.e.,

$$
\left\{\begin{array}{c}
\boldsymbol{\alpha}=-\nabla \times \boldsymbol{\beta}^{p}=\frac{1}{2} \in: \boldsymbol{S} \\
\boldsymbol{\eta}=\nabla \times \nabla \times \boldsymbol{\varepsilon}^{p}=\frac{1}{4 g} \in: \in: \boldsymbol{R}(g=\operatorname{det}(\boldsymbol{g}))
\end{array}\right.
$$

where $\boldsymbol{\beta}^{p}$ and $\varepsilon_{i j}^{p}=\left(\beta_{i j}^{p}+\beta_{j i}^{p}\right) / 2 \equiv \beta_{(i j)}^{p}$ are plastic distortion and strain tensors, respectively, while $\boldsymbol{g}$ (or $g_{i j}$ ) is the metric tensor of the base space considered, whose difference between before and after deformation defines the strain tensor, i.e., $\varepsilon_{i j}=$ $\left(\mathrm{g}_{i j}-\delta_{i j}\right) / 2$. Here, $(\mathrm{O})_{\text {sym }}=\left(\mathrm{O}+\mathrm{\circ}^{T}\right) / 2$ denotes symmetrization process.

As can be understood from the definitions in Equation (1), $\boldsymbol{\alpha}$ and $\boldsymbol{\eta}$ are interrelated as,

$$
\begin{gathered}
\boldsymbol{\eta}=-(\nabla \times \boldsymbol{\alpha})_{\text {sym }} \\
=-\frac{1}{2}\left\{\nabla \times \boldsymbol{\alpha}+(\nabla \times \boldsymbol{\alpha})^{T}\right\} .
\end{gathered}
$$

Furthermore, the incompatibility tensor $\eta$ can be decomposed into the pure rotation part $\boldsymbol{\Theta}$ and the pure deformation part $\nabla \times \boldsymbol{K}^{T}$, as [13]

$$
\eta=\boldsymbol{\Theta}+\nabla \times \boldsymbol{K}^{T}
$$

where $\Theta$ is also referred to as the disclination tensor, while $\boldsymbol{K}$ is Nye's contortion tensor, defined as $\boldsymbol{K}^{T}=-\boldsymbol{\alpha}+\frac{1}{2} I \cdot \operatorname{tr} \boldsymbol{\alpha}$.

One of the key ideas for the above (b), i.e., "evolution” of inhomogeneities, is to regard the incompatibility tensor $\eta$ as the microscopic degrees of freedom (hereafter, $\mu$ DOFs) of underlying sorts, responsible for driving the field evolutions, embodied as the pertaining working hypothesis called flow-evolutionary law (FEL) [7], which is briefly described in the following. 


\subsection{From Classical plasticity through FTMP-based plasticity}

Appropriate microscopic degrees of freedom ( $\mu \mathrm{DOFs}$ ) are generally necessary to be introduced or taken into account in attaining the descriptive capabilities of the theory being used. Figure 2 overviews the progress of development for theory of plasticity, (1) through (3), before reaching (4) the current FTMP in the light of $\mu \mathrm{DOFs}$.

The potential-based classical theory of plasticity [29,30] (row (1)) puts its basis on the yield function that is given as a function of the second invariance of the deviatoric stress tensor, $J_{2} \equiv \boldsymbol{\sigma}^{\prime}: \boldsymbol{\sigma}^{\prime} / 2$, i.e., $f\left(J_{2}\right)=0$, to be further used for defining the associated flow rule, and ultimately, for deriving the elasto-plastic constitutive equation based on it. In this case, $J_{2}$ itself can be regarded as the $\mu \mathrm{DOF}$ for the theory construction. A shortcoming of this framework is the inability to express the plastic anisotropy-related features. A straightforward way to solve this is to explicitly introduce the slip-based KI, in the place of using $J_{2}$, i.e.,

$$
\tau^{(\alpha)}=\boldsymbol{P}^{(\alpha)}: \boldsymbol{\sigma} \text { and } \dot{\varepsilon}^{\prime}=\sum_{(\alpha)} \boldsymbol{P}^{(\alpha)} \dot{\gamma}^{(\alpha)}
$$

with $\tau^{(\alpha)}$ and $\dot{\gamma}^{(\alpha)}$ the resolved shear stress and strain rate, respectively. Here, $\boldsymbol{P}^{(\alpha)} \equiv$ $\left(\boldsymbol{s}^{(\alpha)} \times \boldsymbol{m}^{(\alpha)}\right)_{\text {sym }}$ represents the Schmid tensor for the slip systems with the slip direction (Burgers vector) and slip plane normal $\left(\boldsymbol{s}^{(\alpha)}, \boldsymbol{m}^{(\alpha)}\right)$. Such framework is conventionally called crystal plasticity (CP) (row (2)) [29-31].

One of the deficiencies of the CP include the inability to take account of the absolute size and, thus, to express the associated scale effects. Coping with this, widely-attempted approach includes the use of "strain gradients [32,33]" via, e.g., the density of "geometrically-necessary dislocations (GNDs) [32]" $\rho_{G N}$ (row (3)). Note, the concept of $\rho_{G N}$ is essentially equivalent to the dislocation density tensor $\boldsymbol{\alpha}$ defined in Equations (1-2) in the context of the nonRiemannian plasticity and the current FTMP. The CP, even accommodated with the GNDsbased $\mu \mathrm{DOFs}$, however, is basically incompetent to predict dislocation substructures in general, neither in terms of the final morphologies nor of their evolutionary aspects, simply because of the lack of necessary information for them in the first place.

To all the above, the current FTMP can provide, at least, one of the rational solutions, simply by introducing the incompatibility-related $\mu \mathrm{DOFs}$ in an appropriate manner. The simulated examples presented in Figure 2 eloquently demonstrates that the incompatibilitybased $\mu$ DOFs ( $\eta-\mu D O F s$, hereafter) is indispensable for such inhomogeneous fields to adequately evolve. 
(1)

\section{Macro Plasticity}

$$
\begin{gathered}
f\left(J_{2}\right)=0 \\
\text { Math. Description } \\
\text { via Stress Invariant } \\
\text { DOFs }
\end{gathered}
$$
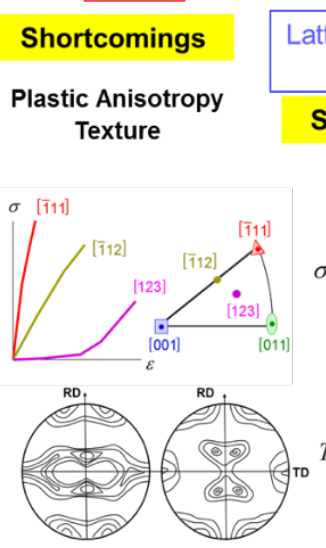

(2)

Crystal Plasticity
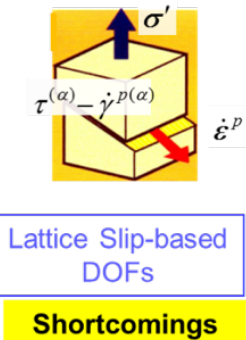

Scale/Size Effects

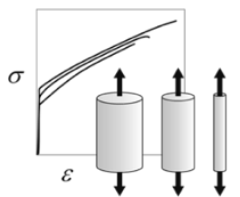

(3)

GN-Crystal Plasticity
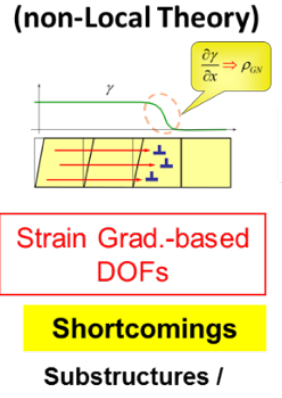

Fracture Modes

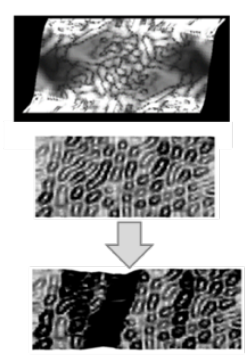

(4)

Field Theory of Multiscale Plasticity

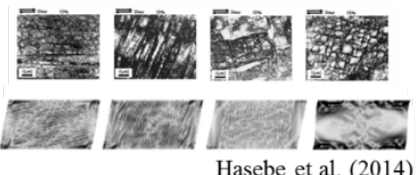

Hasebe et al. (2014)
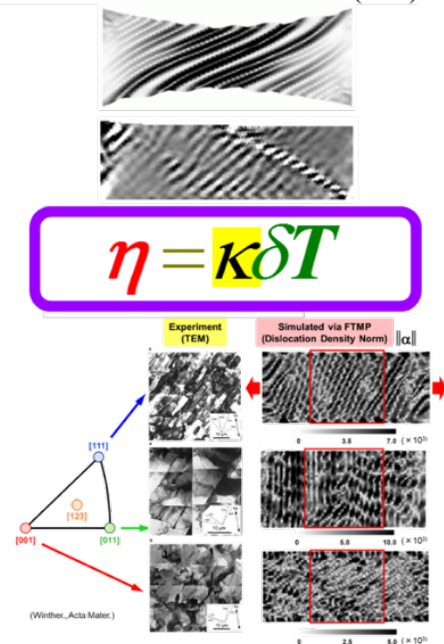

Figure 2: Developmental process of theory of plasticity in the light of $\mu D O F s$, from conventional macro plasticity up to current FTMP-based approach.

\section{KINEMATICS OF CRYSTAL PLASTICITY}

3.1. Kinematics framework of crystal plasticity for finite deformation

We use the extended multiplicative decomposition of the deformation gradient tensor by Lee [34] to include the twinning/kinking mode, within the conventional kinematical framework of the continuum mechanics-based CP [29-31]. By introducing the corresponding intermediate configuration to the twinning (this is same for the kink model in 6), we have,

$$
\boldsymbol{F}=\boldsymbol{F}^{e} \cdot \boldsymbol{F}^{t w} \cdot \boldsymbol{F}^{p}
$$

where $\boldsymbol{F}^{*}$ and $\boldsymbol{F}^{p}$ are the deformation gradient tensors for combined elastic-rigid body rotations and slip-based plastic deformation modes, respectively, while that for the deformation twinning/kinking is all represented together by $\boldsymbol{F}^{t w}$,, to which we introduce the corresponding $\eta$ - $\mu$ DOFs.

The corresponding velocity gradient tensor to the above $\boldsymbol{F}$ is derived based on $\boldsymbol{L}=\dot{\boldsymbol{F}}$. $\boldsymbol{F}^{-1}$, and is further decomposed into the symmetric and skew-symmetric part, i.e., $\boldsymbol{L}=$ $(\boldsymbol{L})_{\text {sym }}+(\boldsymbol{L})_{\text {skew }} \equiv \boldsymbol{d}+\boldsymbol{\Omega}$, where $\boldsymbol{d}$ and $\boldsymbol{\Omega}$ are called deformation rate and spin tensors, respectively [29-31]. With all these, we collectively have, 


$$
\begin{gathered}
\boldsymbol{L}=\dot{\boldsymbol{F}} \cdot \boldsymbol{F}^{-1} \equiv \boldsymbol{L}^{*}+\boldsymbol{L}^{p}+\boldsymbol{L}^{t w} \\
=\left(\boldsymbol{d}^{*}+\boldsymbol{\Omega}^{*}\right)+\left(\boldsymbol{d}^{p}+\boldsymbol{\Omega}^{p}\right)+\left(\boldsymbol{d}^{t w}+\boldsymbol{\Omega}^{t w}\right) \\
\boldsymbol{d} \equiv \boldsymbol{d}^{*}+\boldsymbol{d}^{p}+\boldsymbol{d}^{t w} \text { and } \boldsymbol{\Omega} \equiv \boldsymbol{\Omega}^{*}+\boldsymbol{\Omega}^{p}+\boldsymbol{\Omega}^{t w}
\end{gathered}
$$

and associatively,

$$
\boldsymbol{d}^{*}=\boldsymbol{d}-\left(\boldsymbol{d}^{p}+\boldsymbol{d}^{t w}\right) \text { and } \boldsymbol{\Omega}^{*}=\boldsymbol{\Omega}-\left(\boldsymbol{\Omega}^{p}+\boldsymbol{\Omega}^{t w}\right)
$$

here, $\boldsymbol{\Omega}^{*}$ is referred to as the substructural spin tensor, responsible for the lattice rotations.

Combined the above Equation (6)2 with the crystal plasticity-based expressions, given in Equation (4)2, individually for the plastic and the twin parts, they ultimately yield the following relationship,

$$
\begin{gathered}
\boldsymbol{L}=\left(\boldsymbol{d}^{*}+\boldsymbol{\Omega}^{*}\right)+\left(\boldsymbol{d}^{p}+\boldsymbol{\Omega}^{p}\right)+\left(\boldsymbol{d}^{t w}+\boldsymbol{\Omega}^{t w}\right) \\
\equiv \boldsymbol{d}^{*}+\boldsymbol{\Omega}^{*}+\left(\sum_{(\alpha)} \boldsymbol{P}^{p(\alpha)} \dot{\gamma}^{p(\alpha)}+\sum_{(\alpha)} \boldsymbol{W}^{p(\alpha)} \dot{\gamma}^{p(\alpha)}\right)+\left(\sum_{(\bar{\alpha})} \boldsymbol{P}^{t w(\bar{\alpha})} \dot{\gamma}^{t w(\bar{\alpha})}+\sum_{(\bar{\alpha})} \boldsymbol{W}^{t w(\bar{\alpha})} \dot{\gamma}^{t w(\bar{\alpha})}\right)
\end{gathered}
$$

where $\boldsymbol{P}^{i(\alpha)}=\left(\boldsymbol{s}^{i(\alpha)} \otimes \boldsymbol{m}^{i(\alpha)}\right)_{\text {sym }}$ represents the Schmid tensors for slip and twin modes with $i=p$ or $t w$, respectively, while $\boldsymbol{W}^{i(\alpha)}=\left(\boldsymbol{s}^{i(\alpha)} \otimes \boldsymbol{m}^{i(\alpha)}\right)_{\text {skew }}$ are the material spin tensors, with $(\circ)_{\text {skew }} \equiv\left(\circ-\bigcirc^{T}\right) / 2$ denoting the skew-symmetrization process.

\subsection{Treatments of lattice rotations}

For the slip and twin systems assumed, represented collectively by $\left(\boldsymbol{s}^{i(\alpha)}, \boldsymbol{m}^{t(\alpha)}\right)$ with $i=p$ and/or $i=t w$, they are updated as,

$$
\boldsymbol{s}^{i *(\alpha)}=\boldsymbol{R} \cdot \boldsymbol{s}^{i(\alpha)} \text { and } \boldsymbol{m}^{t *(\alpha)}=\boldsymbol{R} \cdot \boldsymbol{m}^{t(\alpha)}
$$

where $\boldsymbol{R}$ is the orthogonal tensor, responsible for rigid rotations of the lattice that satisfies $\boldsymbol{R}$. $\boldsymbol{R}^{T}=\boldsymbol{I}$, derived out of the deformation gradient tensor $\boldsymbol{F}$ based on the polar-decomposition theorem, i.e., $\boldsymbol{F}=\boldsymbol{R} \cdot \boldsymbol{U}=\boldsymbol{V} \cdot \boldsymbol{R}$, with $\boldsymbol{U}$ and $\boldsymbol{V}$ being the right and left Cauchy-Green stretch tensors, respectively [29,30]. For updating $\boldsymbol{R}$, we utilize the above substructural spin tensor $\boldsymbol{\Omega}^{*}$ as [35],

$$
\Delta \boldsymbol{R}=\boldsymbol{I}+\frac{\sin \left\|\boldsymbol{\Omega}^{*}\right\| \Delta t}{\left\|\boldsymbol{\Omega}^{*}\right\| \Delta t} \boldsymbol{\Omega}^{*} \Delta t+\frac{1-\cos \left\|\boldsymbol{\Omega}^{*}\right\|}{\left(\left\|\boldsymbol{\Omega}^{*}\right\| \Delta t\right)^{2}}\left(\boldsymbol{\Omega}^{*} \Delta t\right)^{2}
$$

where $\Delta t$ is the increment of the time step. 


\subsection{Elasto-plastic constitutive equation}

Since the crystal lattice is not affected by slip and twin-based inelastic deformations, the elastic constitutive equation can be written as,

$$
\stackrel{\nabla}{\boldsymbol{\tau}^{*}}=\boldsymbol{C}^{e}: \boldsymbol{d}^{*}=\boldsymbol{C}^{e}:\left(\boldsymbol{d}^{p}+\boldsymbol{d}^{t w}\right)
$$

where $\boldsymbol{C}^{e}$ is the elastic tensor. Here, $\stackrel{\nabla}{*}$ represents the Jaumann rate of Kirchhoff stress tensor based on the lattice rotations [29-31], defined as the rate of change of Kirchhoff stress $\boldsymbol{\tau}^{*} \equiv$ $\operatorname{det}(\boldsymbol{F}) \boldsymbol{\sigma}$ seen by an observer who rotates with the lattice according to $\boldsymbol{\Omega}^{*}$, i.e., $\boldsymbol{\tau}^{*}=\dot{\boldsymbol{\sigma}}-\boldsymbol{\Omega}^{*}$. $\sigma+\sigma \cdot \Omega^{*}$, where $\boldsymbol{\sigma}$ and $\boldsymbol{\sigma}$ are the Cauchy stress tensor and its material time rate, respectively. The difference of $\stackrel{\nabla}{\boldsymbol{\tau}^{*}}$ and that based on the material spin $\stackrel{\nabla}{\boldsymbol{\tau}}$ yields,

$$
\stackrel{\nabla}{\boldsymbol{\tau}^{*}}-\stackrel{\nabla}{\boldsymbol{\tau}}=\left(\boldsymbol{\Omega}^{p} \cdot \boldsymbol{\tau}-\boldsymbol{\tau} \cdot \boldsymbol{\Omega}^{p}\right)+\left(\boldsymbol{\Omega}^{t w} \cdot \boldsymbol{\tau}-\boldsymbol{\tau} \cdot \boldsymbol{\Omega}^{t w}\right)
$$

in the present context. Combining this with Equation (11) for $\stackrel{\nabla}{\boldsymbol{\tau}^{*}}$, and using the microscopic expressions for $\boldsymbol{\Omega}^{p}$ and $\boldsymbol{\Omega}^{t w}$ in Equation (8), we finally have,

$$
\stackrel{\nabla}{\boldsymbol{\tau}}=\boldsymbol{C}^{e}: \boldsymbol{d}-\sum_{(\alpha)}^{N} \boldsymbol{R}^{p(\alpha)} \dot{\gamma}^{p(\alpha)}+\sum_{(\bar{\alpha})}^{\bar{N}} \boldsymbol{R}^{t w(\bar{\alpha})} \dot{\gamma}^{t w(\bar{\alpha})}
$$

with

$$
\left\{\begin{array} { c } 
{ \boldsymbol { R } ^ { p ( \alpha ) } = \boldsymbol { C } ^ { e } : \boldsymbol { P } ^ { p ( \alpha ) } + \boldsymbol { \beta } ^ { p ( \alpha ) } } \\
{ \boldsymbol { \beta } ^ { p ( \alpha ) } = \boldsymbol { W } ^ { p ( \alpha ) } \cdot \boldsymbol { \tau } - \boldsymbol { \tau } \cdot \boldsymbol { W } ^ { p ( \alpha ) } }
\end{array} \quad \text { and } \quad \left\{\begin{array}{c}
\boldsymbol{R}^{t w(\bar{\alpha})}=\boldsymbol{C}^{e}: \boldsymbol{P}^{t w(\bar{\alpha})}+\boldsymbol{\beta}^{t w(\bar{\alpha})} \\
\boldsymbol{\beta}^{t w(\bar{\alpha})}=\boldsymbol{W}^{t w(\bar{\alpha})} \cdot \boldsymbol{\tau}-\boldsymbol{\tau} \cdot \boldsymbol{W}^{t w(\bar{\alpha})}
\end{array}\right.\right.
$$

\section{CONSTITUTIVE MODELS AND ANALYTICAL CONDITION}

\subsection{FTMP-based model for deformation twinning: First-step model}

In the present series of attempts for reproducing deformation-induced "kink" morphologies, we make use of the FTMP-based model ( $\eta-\mu D O F s)$ as an alternative and/or additional model of inelastic deformation. As the first-step model, we utilize the $\eta-\mu D O F s$ for deformation twinning $[18,19]$, in addition to the major deformation mode by dislocation glides.

In this first-step model, the strain rate due to deformation twinning is assumed to be driven by the evolution of the corresponding incompatibility tensor field to the twinning mode, $\left(\boldsymbol{t}^{t w(\bar{\alpha})} \otimes \boldsymbol{s}^{t w(\bar{\alpha})}\right): \boldsymbol{\eta}$, i.e., 


$$
\begin{gathered}
\dot{\gamma}^{t w(\bar{\alpha})}=Q_{\bar{\alpha} \bar{\beta}}^{t w}\left|\dot{\gamma}_{\text {prev }}^{t w(\bar{\beta})}\right| \\
Q_{\bar{\alpha} \bar{\beta}}^{t}=\left(\mathrm{A}+F\left(\eta_{t w}^{(\bar{\beta})}\right)\right) \cdot\left\langle 1-\frac{\left|F\left(\eta_{t w}^{(\bar{\beta})}\right)\right|}{F_{s a t}}\right)
\end{gathered}
$$

for the twin systems $\left(\boldsymbol{s}^{t w(\bar{\alpha})}, \boldsymbol{m}^{t w(\bar{\alpha})}\right)$, with $\boldsymbol{t}^{t w(\bar{\alpha})} \equiv \boldsymbol{s}^{t w(\bar{\alpha})} \times, \boldsymbol{m}^{t w(\bar{\alpha})}$. Thus, the activation of twinning in the present model depends solely on the evolution of $F\left(\eta_{t w}^{(\bar{\alpha})}\right)$, which denotes the FTMP-based incompatibility term $[7,18,19]$ defined as,

$$
F\left(\eta_{t w}^{(\bar{\alpha})}\right)=\operatorname{sgn}\left(\eta_{t w}^{(\bar{\alpha})}\right) \frac{\bar{k}}{p_{\eta}}\left(\frac{l_{t w i n}}{b}\left|\eta_{t w}^{(\bar{\alpha})}\right|\right)^{1 / 2}
$$

where $\bar{k}$ is a coefficient, while $l_{\text {twin }}$ represents the characteristic length of the twinning field, normally coinciding with the lattice constant.

\subsection{FTMP-based model for slip - major deformation mode}

For the slip modes, we use the constitutive equation derived on the basis of the statistical mechanics-based dislocation dynamics [7,8], which serves a natural expression of the dislocation glide-based crystal plasticity in terms of strain rate/temperature dependency, given as,

$$
\dot{\gamma}^{p(\alpha)}=\dot{A}_{S R} \operatorname{sgn}\left(\tau^{(\alpha)}\right)\left\{\exp B_{S R}\left(1.0-\left|\frac{\tau^{(\alpha)}}{K^{(\alpha)}}\right|^{p}\right)^{q}+C_{S R}\right\}^{-1}
$$

where $\dot{A}_{S R}, B_{S R}$ and $C_{S R}$ are material parameters. Here, $K^{(\alpha)}$ represents the drag stress, responsible for the isotropic hardening, whose time-evolution defines the instantaneous hardening moduli, further expressed as,

$$
\dot{K}^{(\alpha)}=Q_{\alpha \beta} H\left(\gamma_{\alpha}\right)\left|\dot{\gamma}^{p(\alpha)}\right|
$$

where $H\left(\gamma_{\alpha}\right)$ represents the referential instantaneous hardening moduli. The present constitutive model incorporates the contributions of $\alpha^{(\alpha)}$ and $\eta^{(\alpha)}$ through the hardening ratio $Q_{\alpha \beta}$ as,

$$
Q_{\alpha \beta}=\delta_{\alpha \beta}+f_{\alpha k} S_{\beta k}+\delta_{\alpha \beta}\left\{F\left(\alpha^{(\alpha)}\right)+F\left(\eta^{(\alpha)}\right)\right\} \text { (no sum on } \beta \text { ) }
$$

where $f_{\alpha k}$ denotes the dislocation interaction matrix, while $S_{\beta k}$ expresses the history matrix, further given as an increasing function of the plastic work $\left(W_{p}\right)_{(\beta)}^{*}$ done by the effective stress responsible for the on-going dislocation processes, i.e., 


$$
S_{\alpha \beta}=\tanh \left[\frac{\left(W_{p}\right)_{\alpha \beta}^{*}}{\left(W_{p}\right)_{\text {sat }}^{*}}\right] \text { with }\left(W_{p}\right)_{\alpha \beta}^{*}=\delta_{\alpha \beta}\left(\tau^{(\beta)} \cdot \dot{\gamma}^{p(\beta)}\right)
$$

The strain gradient terms in Equation (19), i.e., $F\left(\alpha^{(\alpha)}\right)$ and $F\left(\eta^{(\alpha)}\right)$, on the other hand, are given respectively as [7],

$$
\left\{\begin{array}{l}
F\left(\alpha^{(\alpha)}\right)=\frac{\bar{k}}{p_{\alpha}}\left(\frac{\left|\alpha^{(\alpha)}\right|}{b}\right)^{1 / 2} \\
F\left(\eta^{(\alpha)}\right)=\operatorname{sgn}\left(\eta^{(\alpha)}\right) \frac{\bar{k}}{p_{\eta}}\left(\frac{l_{\text {defect }}}{b}\left|\eta^{(\alpha)}\right|\right)^{1 / 2}
\end{array}\right.
$$

where $p_{\alpha}, p_{\eta}$ are coefficients, while $l_{\text {defect }}$ represents the characteristic (referential) length of the defect field considered, e.g., $l_{\text {defect }}=b$ for expressing dislocation dipoles and $l_{\text {defect }}=$ $1.0 \mu \mathrm{m}$ for dislocation substructures such as cell structures.

For the explicit forms of the instantaneous hardening moduli $H\left(\gamma_{\alpha}\right)$ in Equation (18), we employ here the following phenomenological models, widely used for HCP Mg for the basal and non-basal slips [19], i.e.,

For basal:

$$
H\left(\gamma_{\alpha}\right)=h_{0}
$$

For non-basal:

$$
H\left(\gamma_{\alpha}\right)=h_{0}\left(1-\frac{\tau_{0}}{\tau_{\infty}}\right) \exp \left(-\frac{h_{0} \gamma_{\alpha}}{\tau_{\infty}}\right)
$$

with

$$
\gamma_{\alpha}=\sum_{(\alpha)}\left|\gamma^{(\alpha)}\right|
$$

where $h_{0}, n$ and $\tau_{0}$ are material parameters.

\subsection{Simulation models and analytical conditions}

Figure 3 shows the schematics of analytical model employed in the present series of finite element (FE) analyses (common to both the models), which is divided into $100 \times 100$ crossed triangle elements (totally 40,000 elements with 20,201 nodes). We refer to the compression test in the c-axis direction under die constraint condition denoted in Figure 3(b), considered by Kelley and Hosford [38]. The nodes at the top end are given a total displacement of $15 \mu \mathrm{m}$, corresponding to $15 \%$ compressive strain of the model, at the strain rate of $10^{-3} \mathrm{~s}^{-1}$ in plane strain condition. 
(a)

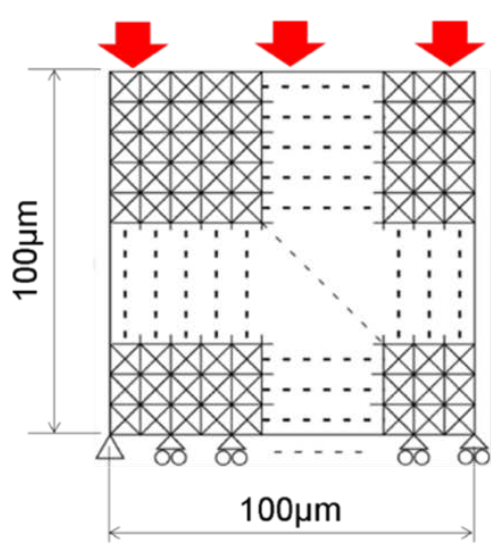

(b)
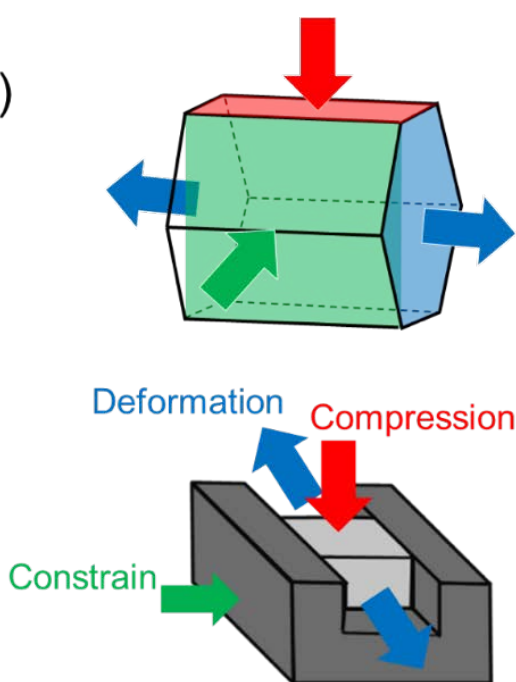

Figure 3: (a) Finite element analytical model. (b) Schematic view of channel-die compression experiment by Kelley and Hosford [38] and single crystal orientation E (Ori. E) assumed in present plane-strain compression simulations.

\section{RESULTS AND DISCUSSIONS: FIRST-STEP MODEL}

\subsection{Basic properties}

The current FTMP-based model accommodated with the $\eta$ - $\mu$ DOFs can reproduce, e.g., a set of orientation-dependent experimental stress-strain curves by Kelly and Hosford [38] for a Mg single crystal [19]. The present model further exhibits not only the commensurate reproducibility of the stress-strain responses with those reported before [19], as presented in Figure 4(a), but also those of the evolving twinned regions explicitly, which is basically absent in the former model. Figure 4(b) shows an example of the growing twinned regions via $\gamma_{t w}$, the corresponding incompatibility via $F\left(\eta^{t w}\right)$ and the attendant rotational field contours for the orientation $\mathrm{A}$, together with the variation of the relative activity of the assumed twin system with strain, in comparison with those for the slip modes. Here, the compressive twinning was started to grow at around $\varepsilon=0.02$, triggering abrupt changes in the activities of the pyramidal and prismatic slips, after which the twinned regions distinctly emerge in the contours. 
(a)

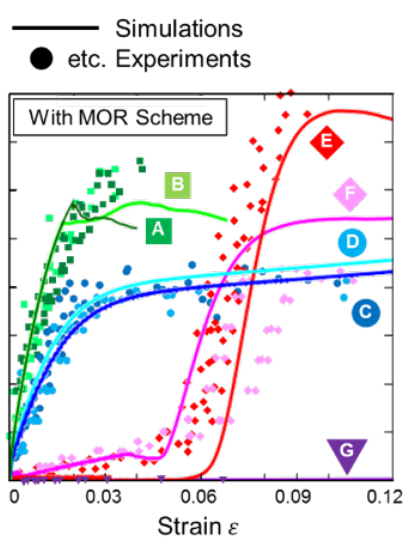

(b)
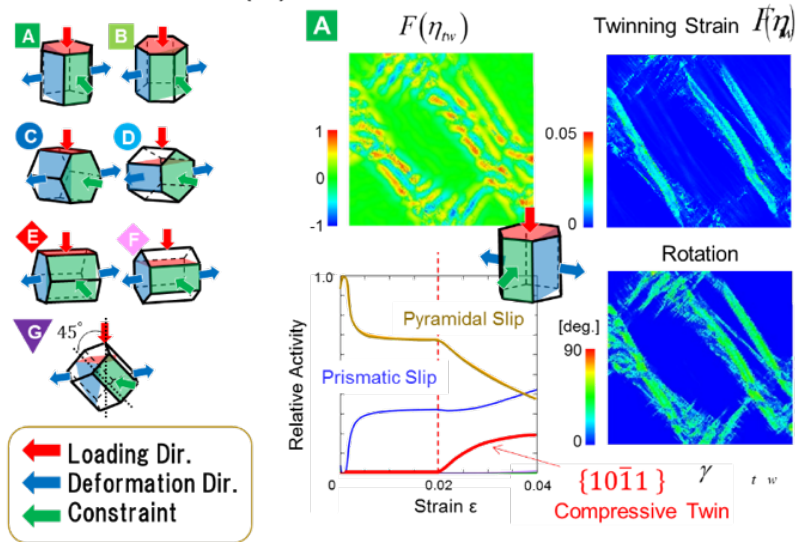

Simulated twinned regions for Ori.A

Figure 4: Simulated orientation-dependent stress-strain curves for Mg single crystals under plane-strain compression (a), over-plotted on corresponding experimental data by Kelley and Hosford [38], together with an example of simulated twinned regions for Ori.A (b), comparing contours of incompatibility, twinning strain and attendant rotation angle, evolved after onset of $\{101 \overline{1}\}$ compressive twinning, together with variation of relative activity of it in comparison with those of pyramidal and prismatic slips.

\subsection{Simulation results: First-step model}

Figure 5(a) presents simulation results for the first step model, showing evolving incompatibility contours and the correspondingly developing rotational fields. The result shows ubiquitous interior initiations and the subsequent growths of "kink-like "regions accompanied by lattice rotations, with rather fixed vertex angle, because of the well-defined twin systems assumed in the simulation. Figure 5(c) shows the nominal stress-nominal strain curves corresponding to the evolving contours, together with the relative activity ratios among the slip (basal, prismatic and pyramidal) and the twin (tensile and compressive) systems. Predominant activities of the tensile twin from the start of deformations are confirmed, resulting in small work hardening rate on the stress-strain curves. They are followed by relatively clear onsets of the compressive twin, respectively, bringing about an increase in the hardening rate. The onset of compressive twinning takes place after 0.08 nominal strain, around which avalanche-like rapid expansions of the "kinking" regions are observed in Figure 5(a). We refer to the morphology of thus simulated regions as "kink-like" hereafter, for making distinction from those obtained for the second-step model below, with which we reproduce much closer "kink" morphologies to the experimental observations. 
(a)
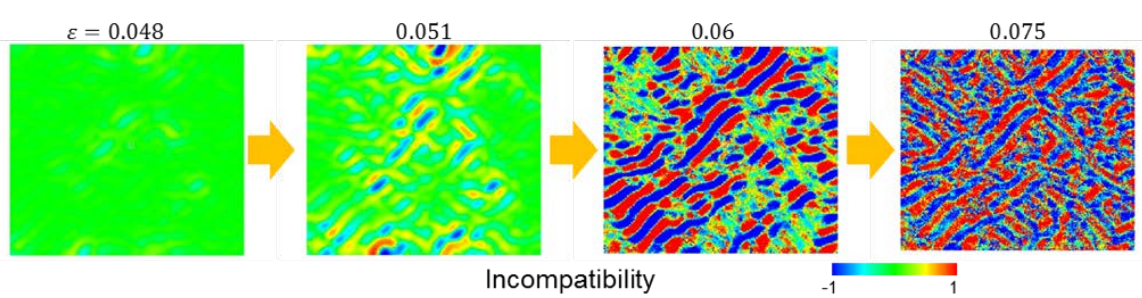

(b)

Incompatibility
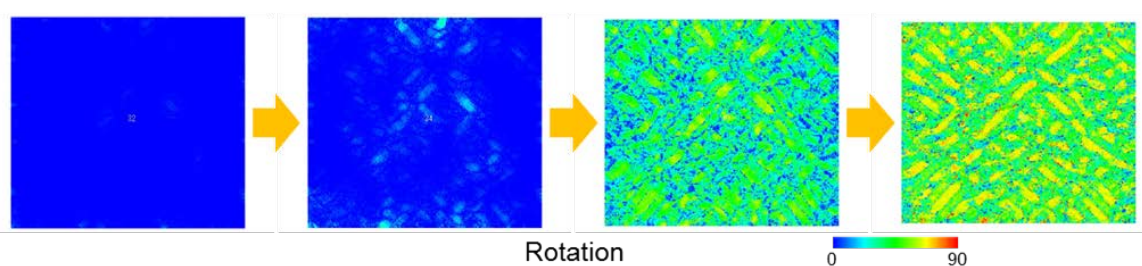

(c)
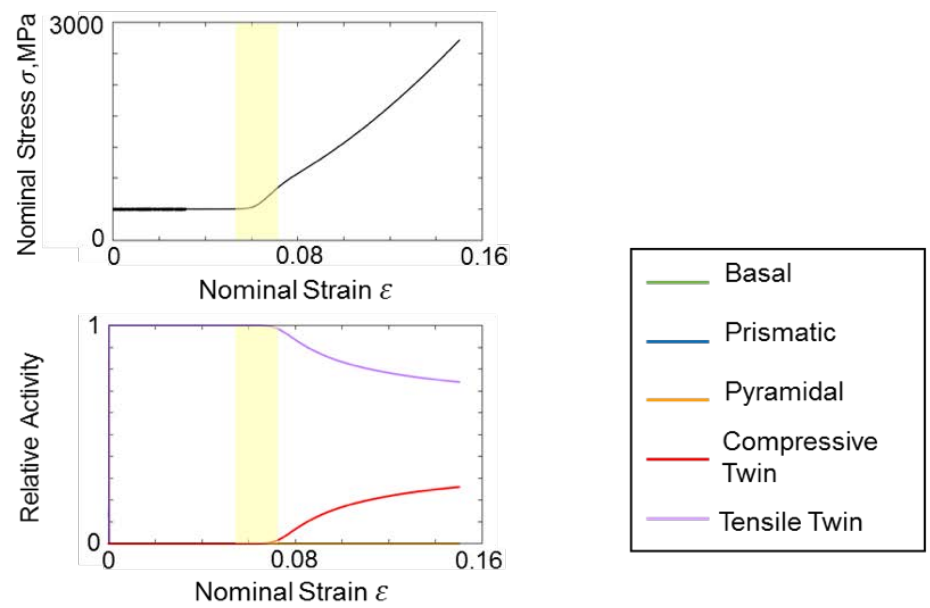

Figure 5: Variations of contour diagrams with strain of (a) incompatibility (via $F\left(\eta_{t w i n}\right)$ and (b) the corresponding rotation (b) for the first-step model, together with (c) nominal stress-nominal strain curve and the associated relative activity ratios.

Aizawa et al. [6] reported rather "anomalous" energy characteristics for the kinking phenomena based on the $\mathrm{AE}$ technique, as presented in Figure 6, where frequency distributions of the measured AE absolute energy distribution (a), comparing between before (i.e., precursor) and after kink formations are confirmed, together with the corresponding return maps against the AE energy difference. As in (a), the AE energy exhibits "scale-free" distribution except the low energy region which yields a flat component. The return map in Figure 6(b) suggests that there exist a strong correlation between the kink formation intervals and the energy released. 
These results demonstrate at least three following features, i.e., (1) relatively larger number of small kinks compared with large kinks (102 difference in size and 106 difference in the energy) are formed, (2) no subsequent formations of large kinks judged from the return maps (b), and substantially the same mechanism can apply both to the precursor and the kink regions.

(a)

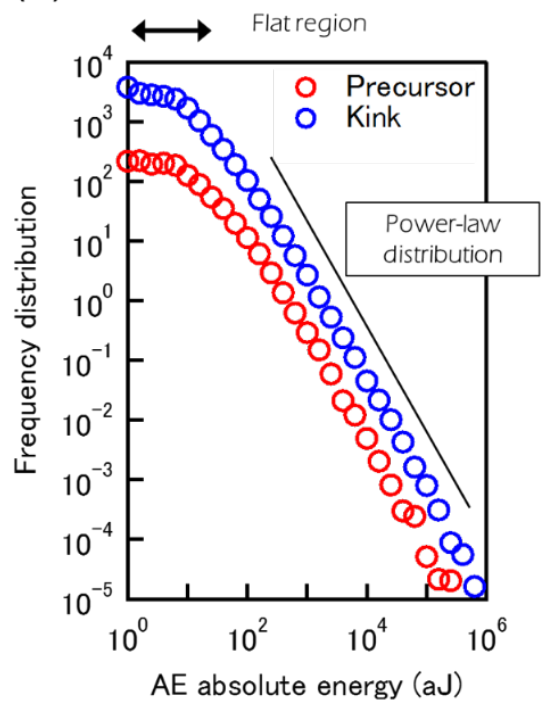

(b)

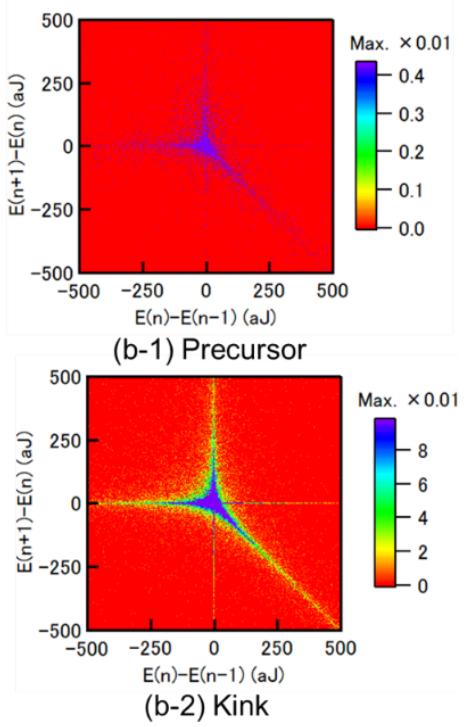

Figure 6: Observed AE energy characteristics by Aizawa et al. (a) Frequency distribution of $A E$ absolute energy (b) Return map diagrams against $A E$ signal energy difference. (Courtesy of Dr. K. Aizawa, JAEA (2018))

Let us examine the attendant energy releasing characteristics for the simulated results (the first-step model). Figure 7 shows a frequency diagram of the elastic strain energy fluctuation $\delta U^{e}$, at four steps of nominal strains, corresponding to those in Figure 5. As demonstrated, the result basically exhibits the similar power-law type distributions to the experiment (Figure 6(a)) from the early stage of deformation, even before the massive emergence of the "kinklike" regions take place. These trends are analogous to the above-mentioned AE-based observations. From this power-law type distribution, we can see scale free characteristics of kinking as this picture implies. In this context, scale free characteristics mean the low probability of growths of large kinks, together with higher probability of small kink growths. 


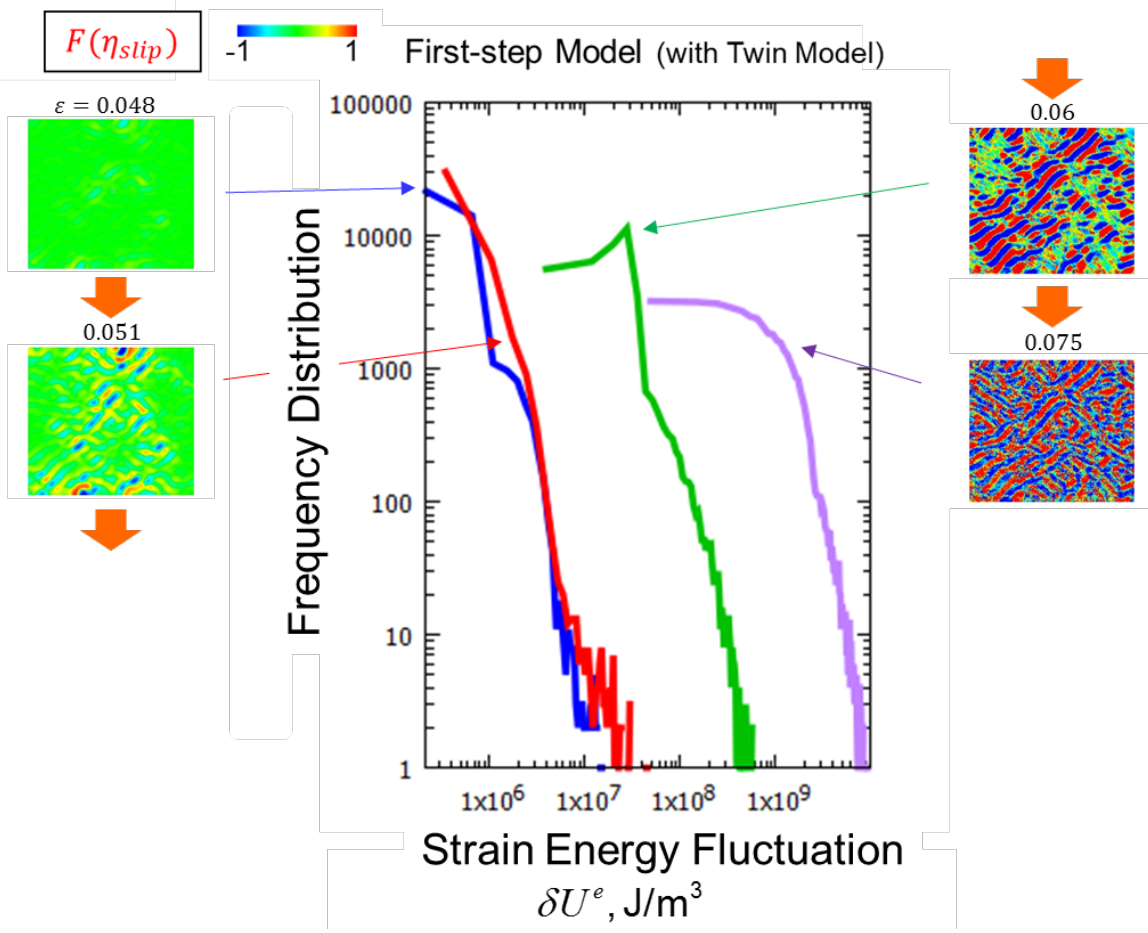

Figure 7: Frequency distribution diagrams of fluctuation of $\delta U^{e}$ for the first-step model.

We also correlate the return maps of $\delta U^{e}$ based on the first-step simulation results in Figure 8 , taking the difference between the temporarily-adjacient values of the obtained elastic strain energy fluctuation, regarding it in here as the energy gap. As indicated in Figure 8, thus obtained distribution of return maps tends to concentrate in the first quadrant, whose trend is different from the experiment in Figure 6(b), exhibiting the clear concentrations in the fourth quadrant for both the precursor (b-1) and the kink (b-2). The first-quadrant concentration in the simulation results can be interpreted as that the energy gaps are constantly positive, meaning a monotonic growths of the size of deformation-induced events (in this case, twinning), as schematically illustrated in Figure 8(b). Micrographical observations, as in the inset in Figure 8, are also negative against these results. 
(a)
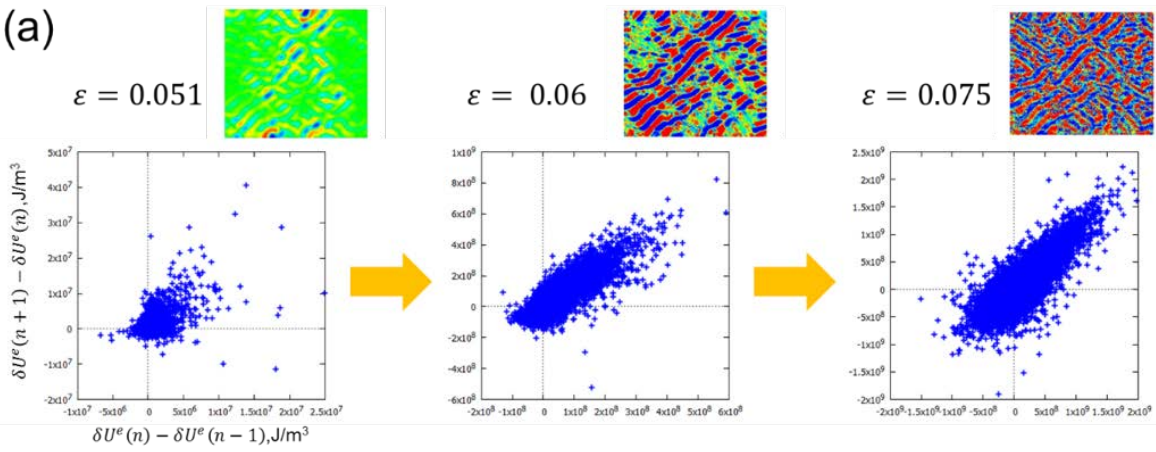

(b)
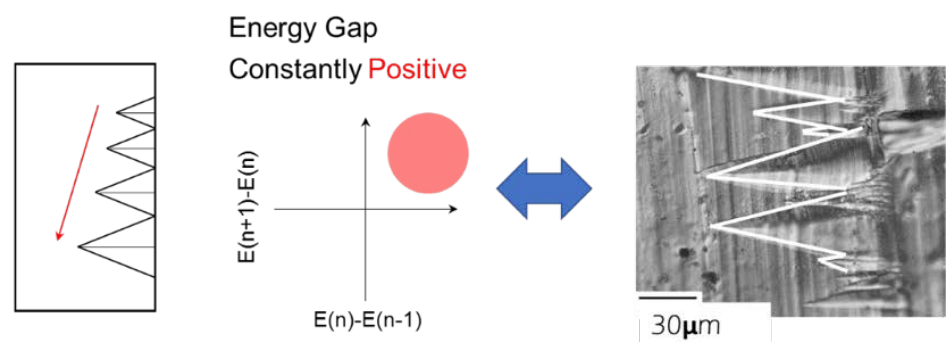

Figure 8: Return maps at three steps from simulated results (a). Relationship between pattern of kink growth and distribution of return map in the first quadrant (b), in comparison with experimental micrograph [3].

\section{KINK MODEL: SECOND-STEP MODEL}

In the place of the twinning model for the first step, we newly introduce the rank-1 connectionbased kink systems into Equations (14) and (15) as the alternative $\eta-\mu D O F s$, which is used here as the second-step model, otherwise basically unaltered.

\subsection{FTMP-based model for kinking}

The condition for the rank-1 connection is represented by [39],

$$
\boldsymbol{F}-\boldsymbol{G}=\boldsymbol{a} \otimes \boldsymbol{n}
$$

where, $\boldsymbol{F}$ and $\boldsymbol{G}$ are deformation gradients, while $\boldsymbol{a}=(\boldsymbol{F}-\boldsymbol{G}) \cdot \boldsymbol{n}$, which ensures the continuity of the deformation on the plane $\boldsymbol{n}$. By applying the rank-1 compatibility condition to the kink band [39], the direction of interface between the kink band and the matrix is defined as shown in Figure 9. Here, the vector $\mathrm{s}$ indicates the deformation direction, while the vector $\mathrm{m}$ is perpendicular to $\mathrm{s}$. Also, the value of $\mathrm{t}$ indicates the magnitude of the shear. 
Referring to the result of application of rank-1 connection to the kink band, we define afresh the $\boldsymbol{s}$ and $\boldsymbol{m}$ vectors in Equation (9), respectively, as,

$$
\begin{gathered}
\boldsymbol{s}=\frac{1}{\sqrt{4+t^{2}}}\left\{\begin{array}{c} 
\pm 2 \\
t^{2} \\
0
\end{array}\right\} \\
\boldsymbol{m}=\frac{1}{\sqrt{4+t^{2}}}\left\{\begin{array}{c} 
\pm t \\
2 \\
0
\end{array}\right\}
\end{gathered}
$$

In the present study, the value of $t$ in Equations (26) and (27) is simply assumed to increase monotonically according to the simulation time steps, common to all the FE elements. Also, as show in Figure 9(b), the $s$ and $m$ vectors change depending on the t value.
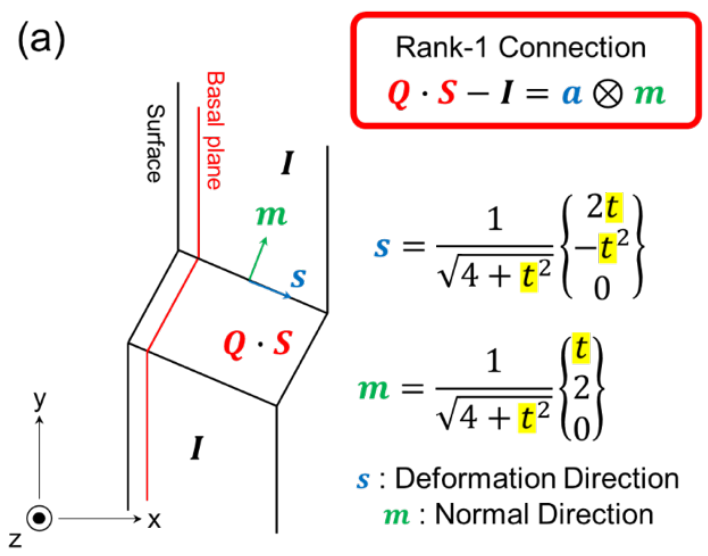

(b)

Figure 9: Schematics of Rank-1 kinematical compatibility condition for a kink band (a), together with analytical condition for the kink model used (b).

The kink model used here is basically the same as the twin model for the first-step analyses (Equations (14) and (15)), except the projection direction of the incompatibility tensor, given as,

$$
\dot{\gamma}^{\operatorname{kink}(\bar{\alpha})}=Q_{\bar{\alpha} \bar{\beta}}^{k i n k}\left|\dot{\gamma}_{\text {prev }}^{\operatorname{kink}(\bar{\beta})}\right|
$$


with

$$
Q_{\bar{\alpha} \bar{\beta}}^{k i n k}=\left(1+F\left(\eta_{\text {kink }}^{(\bar{\beta})}\right)\right) \cdot\left\langle 1-\frac{\left|\alpha\left(\eta_{\text {kink }}^{(\bar{\beta})}\right)\right|}{F_{\text {sat }}}\right\rangle
$$

here, we also introduce the dislocation density term $F\left(\alpha_{\text {kink }}\right)$ in the attenuation term, in the place of $F\left(\eta_{\text {kink }}\right)$, in order to reflect the effect of partial wedge disclination [39].

Figure 10 summarizes the comparison between the twin and current kink models for the first and second-step analyses, emphasizing the difference between the assumed twin and the kink systems represented by s and $\mathrm{m}$.

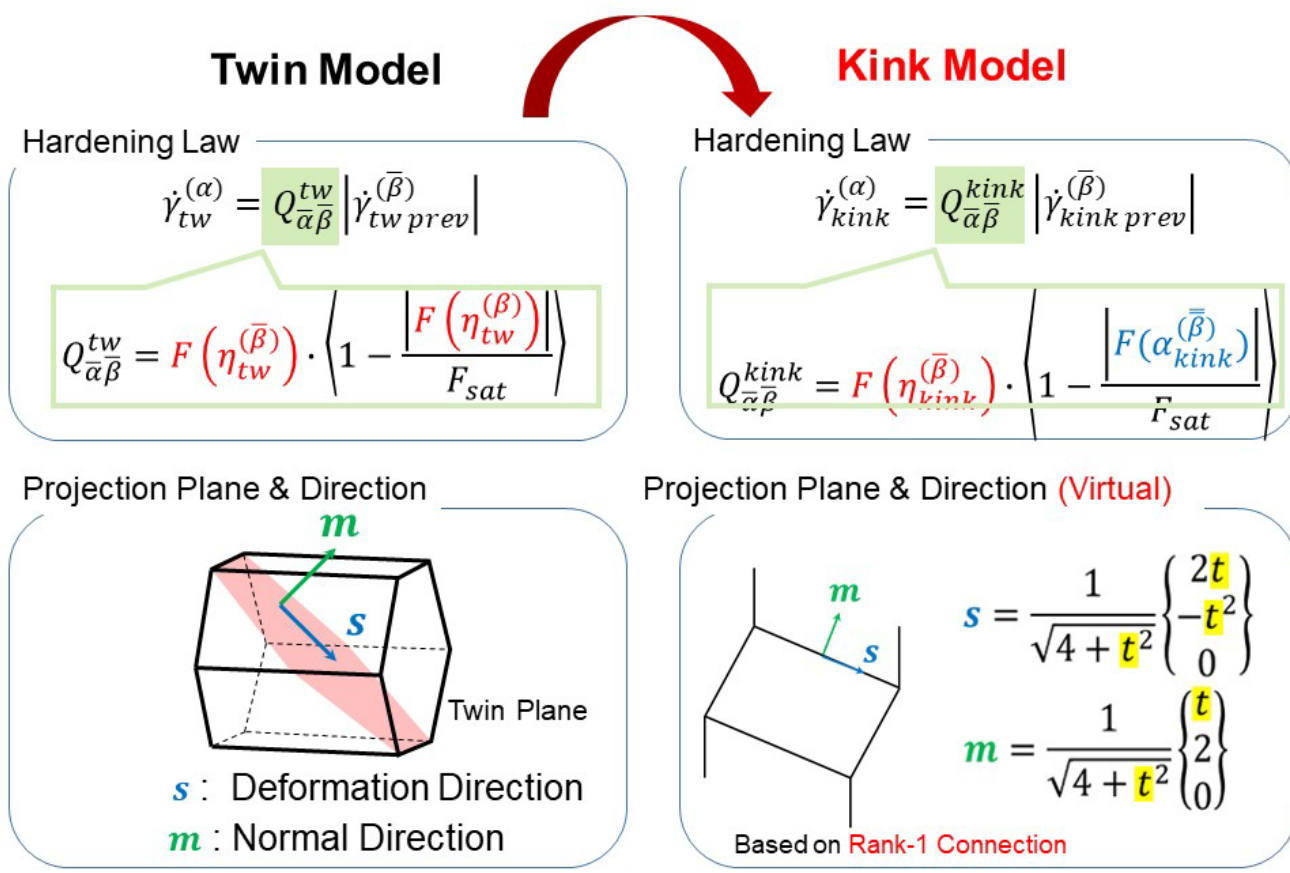

Figure 10: Comparison between twin and kink models for the first and the secondstep analyses.

\subsection{Simulation models and analytical conditions: Second-step model}

Same as the first-step analysis, the simulation model is divided into $100 \times 100$ crossed triangle elements. To mimic a directionally-solidified (DS) single crystal Mg with LPSO mill-feuille structure, we set aligned soft and hard layers in parallel to the basal plane, with $1 \mu \mathrm{m}$ layer widths. The soft/hard regions are controlled by the values of the hardening ratio. Also, we assume that the kink mode is only active, while the basal, prismatic and pyramidal slip and the twin systems are not operative associated with the layered structure. 
(a)

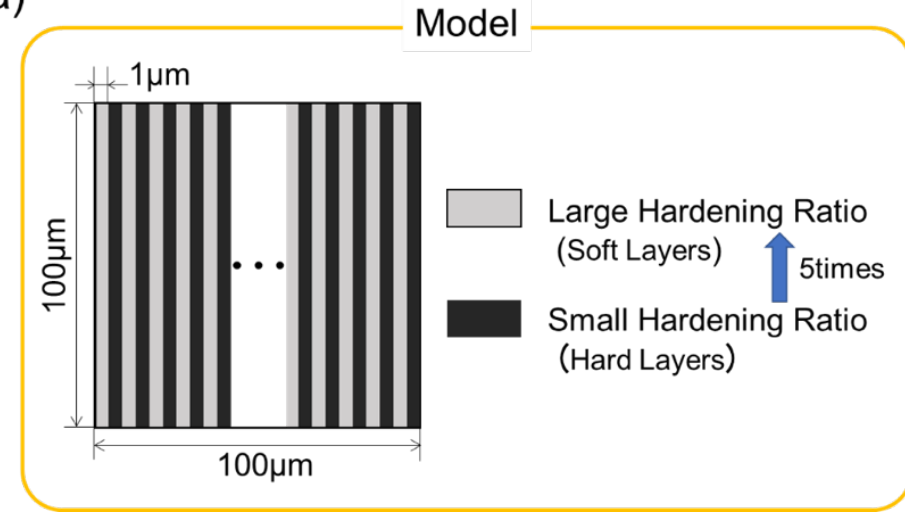

(b)

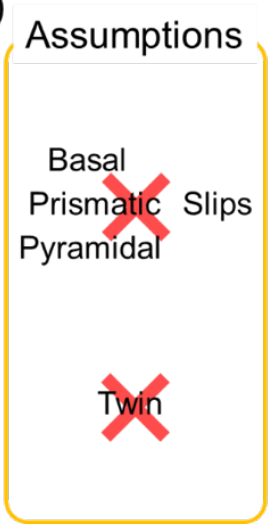

Figure 11: (a) Single crystal Mg model with alternating soft and hard layers, distinguished by the values of the hardening ratio. (b) Assumptions in this study.

\subsection{Results and discussions: Second-step model}

Figure 12 displays the simulated results, showing variation of incompatibility contours with strain (a) and the corresponding rotation angles, measured by the change in the basal plane angle (b). A comparison with a typical micrograph for kinked structure [5] is also made in Figure 12(c), taking the rotational angle counter at $\varepsilon=0.15$ as an example. We confirm successfully reproduced kink morphologies by the present FTMP-based FE simulation, together with the attendant significant misorientation developments, also commensurate with experiments.

The major difference between the current kink (second-step) model and the first-step counterpart (twin model) is in the assumed projection directions of the incompatibility tensor, i.e., s and m, as emphasized in Figure 10. Therefore, we tentatively conclude that one of the necessary conditions for the deformation-induced kink morphologies to be numerically reproduced is a crystallographically-compatible but variable projection directions for the incompatibility tensor, which will possibly be regard as, at least, one of the indispensable microscopic degrees of freedom ( $\mu \mathrm{DOF}$ ) for kink formations.

Figure 13 shows frequency diagrams at four simulation time steps from simulated results. Similar to the first-step (twin) model presented in Figure 7, the result also basically exhibits power-law type distributions from the early stage of deformation, even before the massive emergence of the kink-like regions take place, in good agreement with the experimentallyobserved trends in Figure 6. 
(a)
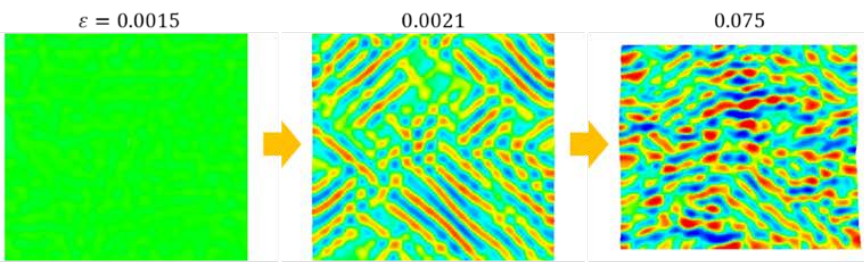

0.15

(b) Incompatibility

$-1 \quad 1$
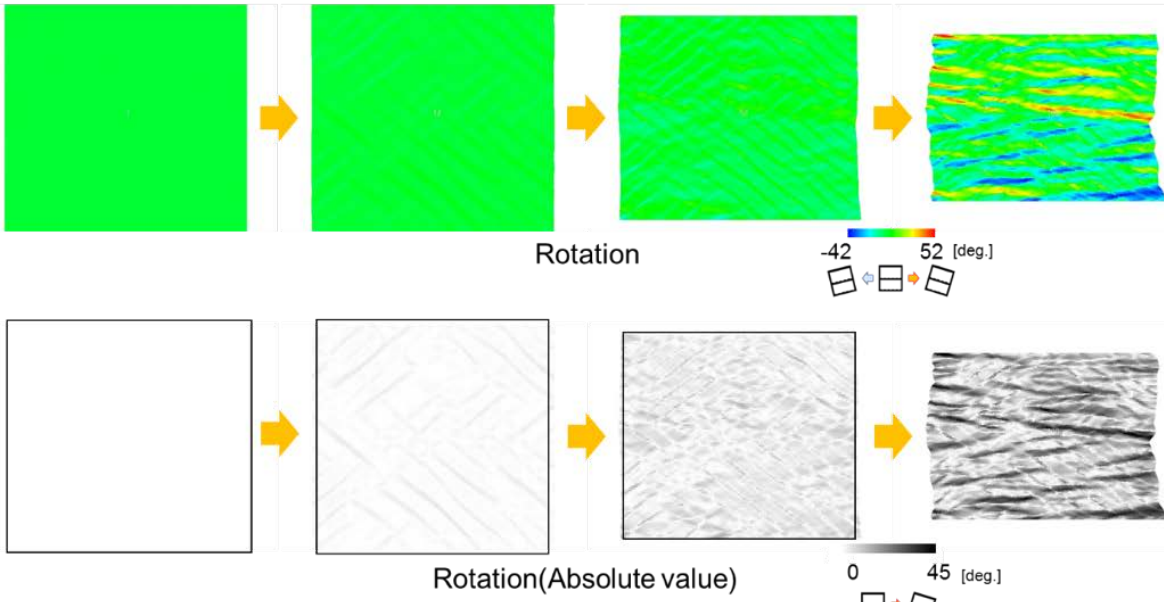

(c)

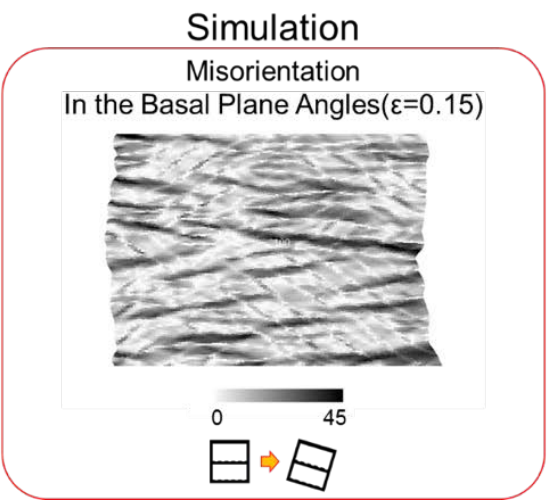

Experiment

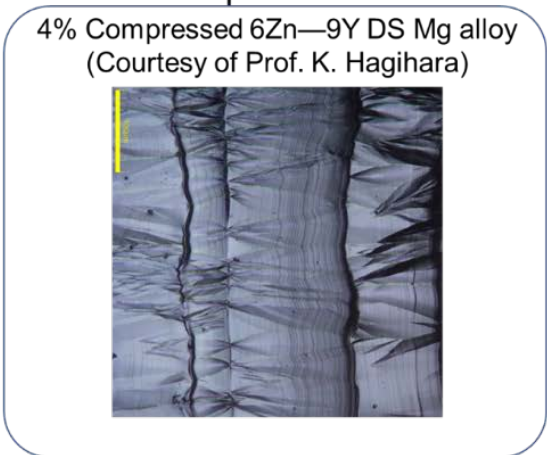

Figure 12: Variations of contour diagrams with strain of (a) incompatibility (via $\left.\mathrm{F}\left(\eta_{\text {kink }}\right)\right)$ and (b) the corresponding rotation (b) for the first-step model, together with a tentative comparison with a micrographically-observed typical kink structure in LPSO-Mg (Courtesy of Prof. K. Hagihara). 


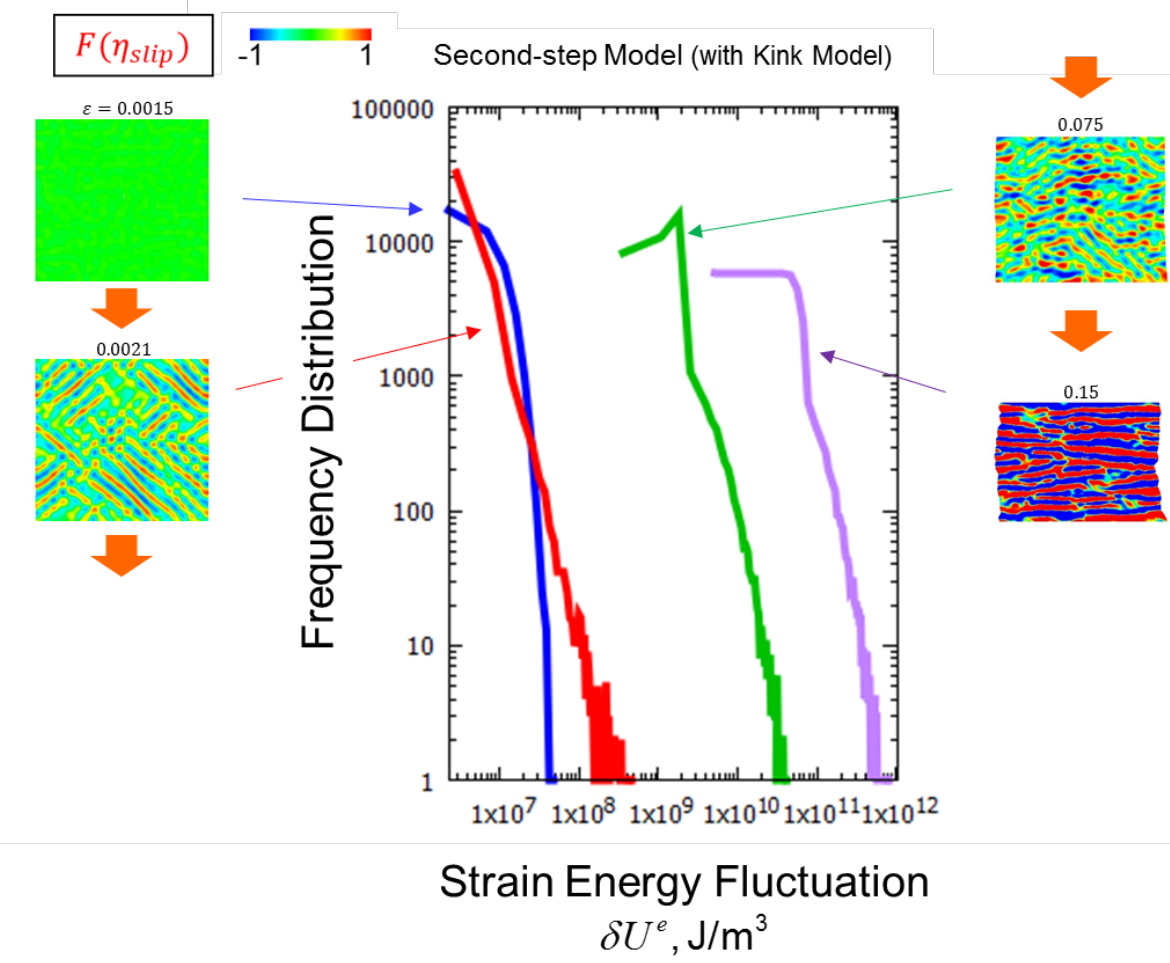

Figure 13: Frequency distribution diagrams of fluctuation of $\delta U^{e}$ for second-step model (with kink model).

Figure 14(a) shows the return maps at three simulation time steps for the current secondstep (kink) model. We observe certain fractions of the elements belonging to the fourth quadrant of the maps, in sharp contrast to those for the first-step model in Figure 8(a). The result is analogous to the AE-based observation in Figure 6(b), meaning that the introduction of the rank-1 connection-based kink system significantly improves the accuracy of the model, in terms not only of the simulated morphology but also the energy releasing characteristics. The existence of the fourth quadrant elements in the current simulation result implies the alternating growths of the large and small kink regions are also successfully reproduced.

This still-incomplete but partially-reproduced energy characteristics can be a strong leverage for supporting the above tentative conclusion, i.e., the incompatibility tensor field coupled with crystallographically-compatible-but-variable projection directions (i.e., appropriate kink systems) is one of the crucial $\mu \mathrm{DOFs}$ for such kink morphologies to be numerically reproduced. 

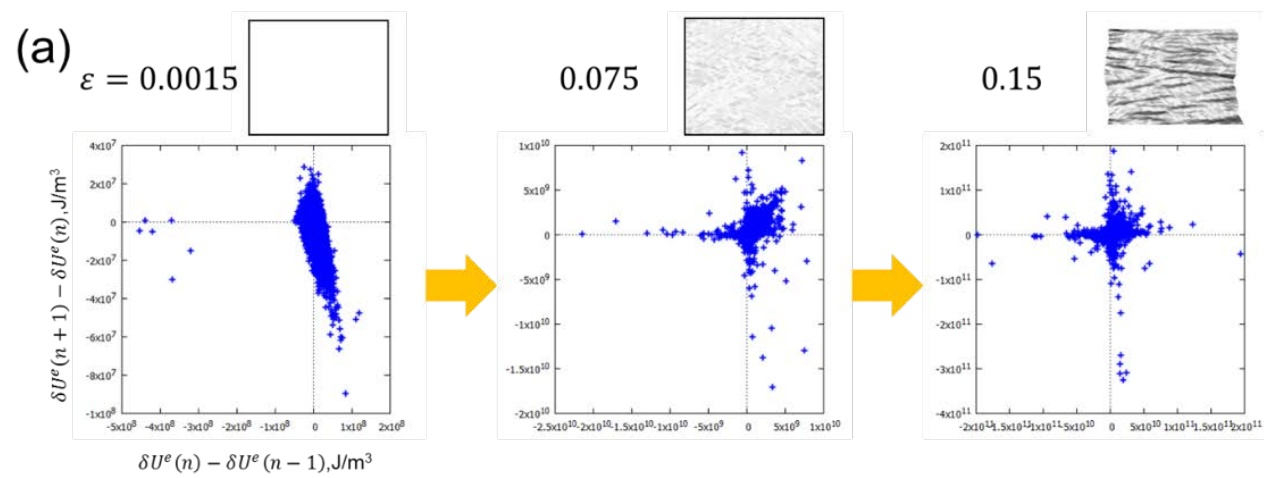

(b)
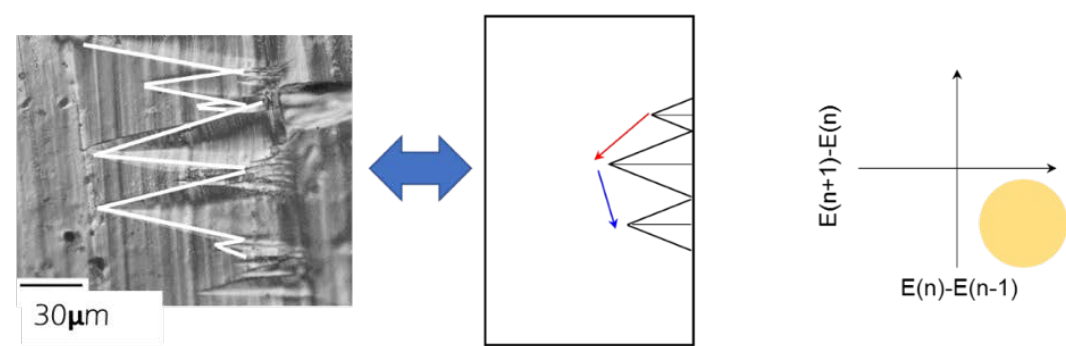

Figure 14: (a)Return maps at three steps from simulated results. (b) Relationship between pattern of kink growth and distribution of return map in the fourth quadrant, in comparison with experimental micrograph [3].

\section{CONCLUSIONS}

In this paper, the FTMP (field theory of multiscale plasticity)-based twin and kink models are utilized to examine the basic capabilities for reproducing the deformation-induced "kink" morphologies. Our main conclusions are as follows.

(1) We succeeded in reproducing kink morphologies, together with the attendant welldeveloped misorientation fields, based on the FTMP-implemented crystal plasticity FE simulation, considering Rank-1 connection for assuming the kink systems. The use of the deformation twin model, on the other hand, also allowed us to simulate "kink-like" structures of evolving kind.

(2) Both twin and kink models exhibited basically mutually-similar similar "scale-free" frequency distributions of strain energy fluctuation both before and after the onset of "kink" region emergence, whose trend is in good agreement with the AE measurements in experiment.

(3) The use of the rank-1 connection-based kink model was demonstrated also to be able to qualitatively reproduce another key feature observed in the return maps for the AE signal energy difference in experiment, whereas the twin model cannot. 
(4) Taking account of all the results above, we tentatively conclude that the appropriate use of the incompatibility tensor is one of the microscopic degrees of freedom $(\mu \mathrm{DOFs})$ for both the "kink" morphologies and the attendant energy release characteristics altogether to be reproduced based on crystal plasticity-implemented finite element simulations, provided the crystallographically-compatible-but-variable kink systems as the projection directions.

\section{ACKNOWLEDGMENTS}

This work was supported by JSPS KAKENHI for Scientific Research on Innovative Areas MFS Materials Science (Grant Number JP18H05483).

\section{REFERENCES}

[1] Kawamura, Y., Hayashi, K., Inoue, A. and Masumoto, T., Rapidly Solidified Powder Metallurgy Mg97Zn1Y2Alloys with Excellent Tensile Yield Strength above $600 \mathrm{MPa}$, Mater. Trans., 2001. 42: p. 1172-1176.

[2] Yamasaki, M., Hashimoto, K., Hagihara, K. and Kawamura, Y., Effect of multimodal microstructure evolution on mechanical properties of Mg-Zn-Y extruded alloy, Acta Mater., 2011. 59: p. 3646-3658.

[3] Hagihara, K., Yokotani, N. and Umakoshi, Y., Plastic deformation behavior of Mg12YZn with 18R long-period stacking ordered structure, Intermetallics., 2010. 18: p. 267-276.

[4] Abe, E., Ono, A., Itoi, T., Yamasaki, M. and Kawamura, Y., Polytypes of long-period stacking structures synchronized with chemical order in a dilute Mg-Zn-Y alloy, Philos. Mag. Lett., 2011. 91: p. 690-696.

[5] Hagihara, K., Private Communication, 2018.

[6] Aizawa, K., Private Communication, 2018.

[7] Hasebe, T., Sugiyma, M., Adachi, H., Fukutani, S. and Iida, M., Modeling and Simulations of Experimentally-Observed Dislocation Substructures Based on Field Theory of Multiscale Plasticity (FTMP) Combined with TEM and EBSD-Wilkinson Method for FCC and BCC Poly/Single Crystals, Mater. Trans., 2014. 55(5): p. 779-787.

[8] Hasebe, T., Kumai, S. and Imaida, Y., Impact compression behavior of FCC metals with pre-torsion strains, Jnl. Maters. Process. Technol., 1999. 85: p. 184-187.

[9] Hasebe, T. and Imaida, Y., Construction of Quantum Field Theory of Dislocations based on the Non-Riemannian Plasticity, Acta Metall. Sin., 1998. 11(6): p. 405-411.

[10] Hasebe, T., Continuum Description of Inhomogeneously Deforming Polycrystalline Aggregate based on Field Theory, IUTAM Symposium on Mesoscopic Dynamics of Fracture Process and Materials Strength. Eds. H. Kitagawa and Y. Shibutani, Kluwer Academic Publishers, 2004: p. 381-390.

[11] Hasebe, T., Field Theoretical Multiscale Modeling of Polycrystal Plasticity, Trans. MRSJ, 2004. 29: p. 3619-3624. 
[12] Hasebe, T., Multiscale Crystal Plasticity Modeling based on Field Theory, Comp. Mech. Eng. Sci. (CMES), 2006. 11: p. 145-155.

[13] Aoyagi, Y. and Hasebe, T., New Physical Interpretation of Incompatibility Tensor and Its Application to Dislocation Substructure Evolution, Key Materials Engineering, 2007. 340(341): p. 217-222.

[14] Yamada, M., Hasebe, T., Tomita, Y., Onizawa, T., Reproducing Kernel Based Evaluation of Incompatibility Tensor in Field Theory of Plasticity, IMMIJ (Interaction and Multiscale Mechanics: An Int. J.), 2008. 1(4): p.437-448.

[15] Hasebe, T., Interaction Fields Based on Incompatibility Tensor in Field Theory of Plasticity-Part I: Theory-, IMMIJ (Interaction and Multiscale Mechanics: An Int. J.), 2009. 2(1): p. 1-14.

[16] Hasebe, T., Interaction Fields Based on Incompatibility Tensor in Field Theory of Plasticity -Part II: Application-, IMMIJ (Interaction and Multiscale Mechanics: An Int. J.), 2009. 2(1): p. 15-30.

[17] Fukutani, S. and Hasebe, T., Extended FTMP to Finslerian Space and Its Application to Modeling and Simulation of Soft Active Materials, Proc. 25th CMD (CMD2012), JSME, 2012: p. 394-395.

[18] Okuda, T., Imiya, K. and Hasebe, T., FTMP-based Simulation of Twin Nucleation and Substructure Evolution under Hypervelocity Impact, Int. J. Comput. Maters. Sci. Eng., 2013. 2(3\&4): p. 1350021.

[19] Kajiwara, N., Imiya, K. and Hasebe, T., FTMP-based Modeling and Simulation of Magnesium, Int. J. Comput. Maters. Sci. Eng., 2013. 2(3\&4): p. 1350022.

[20] Hasebe, T. and Naito, T., FTMP-based 4D Evaluations of Discrete Dislocation Systems, New Frontiers of Nanometals, Eds. S. Faester, et al. (Proc. 35th Ris $\varnothing$ int. Symp. on Maters. Sci.), 2014: p. 305-312.

[21] Ihara, S. and Hasebe, T., FTMP-based simulations and evaluations of GeometricallyNecessary Boundaries (GNBs) of dislocation, Int. Jnl. of Multiphysics, 2019. 13(3): p. 253-268.

[22] Matsubara, Y. and Hasebe, T., Multiscale Modeling and Simulations of Creep Rupture Process of Lath Martensite Block/Packet Structures for High Cr Steels based on FTMP, Proc. 10th Japan-China Bilateral Symp. High Temp. Strength of Maters., 2019: p. 311316.

[23] Kondo, K., Non-Riemannian Geometry of Imperfect Crystals from a Macroscopic Viewpoint, RRAG Memoirs, 1955. 1(D-I): p. 458-469. (RAAG Memoirs of Unifying Study of Basic Problems in Engineering and Physical Science by Means of Geometry (ed: Kondo. K.), Gakujutsu Bunken Fukyu-kai, Tokyo).

[24] Amari, S., A Theory of Deformations and Stresses of Ferromagnetic Substances by Finsler Geometry, RAAG Memoirs, 1962. 3: p. 257-278.

[25] Eshelby, J.D., The force on an elastic singularity, Phil. Trans. Roy. Soc. London SeriesA, 1951. 244(877): p. 87-112. 
[26] Mura, T., Micromechanics of Defects in Solids, 1987: Martinus Nijhoff Publ.

[27] Kadic, A. and Edelen, D.G.B., Lecture Notes in Phys.174, 1983: Springer.

[28] McDavid, A.W. and McMullen, C.D., Generalizing Cross Products and Maxwell's Equations to Universal Extra Dimensions, 2006: http://arxiv.org/abs/hep-ph/0609260.

[29] Khan, A. and Huang, S., Continuum Theory of Plasticity, 1995: Wiley.

[30] Nemat-Nassar, S., Plasticity: A Treatise on Finite De-formation of Heterogeneous Inelastic Materials, 2004: Cambridge Univ. Press.

[31] Asaro, R. J., Micromechanics of Crystals and Polycrystals, Adv. Appl. Mech., 1983. 23: p. 1-115.

[32] Fleck, N.A., Muller, G.M., Ashby, M.F. and Hutchinson, J.W., Strain gradient plasticity: Theory and experiment, Acta Metall, Mater., 1994. 42(2): p. 475-487.

[33] Zbib, H.M. and Aifantis, E.C., On the localization and postlocalization behavior of plastic deformation, Res. Mech., 1988. 23: p. 261-305.

[34] Lee, E.H., Elastic-Plastic Deformation at Finite Strains, J. Appl. Mech., 1969. 36(1): p. $1-6$.

[35] Bassani, J.L. and Wu, T., Latent hardening in single crystals, part II, Analytical characterization and predictions, Proc. R. Soc. A, 1991. 435(1893): p. 21-41.

[36] Kushima, H., Kimura, K. and Abe, F, Degradation of Mod. 9Cr-1 Mo Steel during Longterm Creep Defornation, Tetsu to Hagane, 1999. 85(11): p. 841-847.

[37] Maruyama, K., Sawada, K. and Koike, J., Strengthening Mechanisms of Creep Resistant Tempered Martensitic Steel, ISIJ International, 2001. 41(6): p. 641-653.

[38] Kelley, E.W., and Hosford, W.F., Plane-Strain Compression of Magnesium and Magnesium Alloy Crystals, Trans. Metall. Soc. AIME., 1968. 242: p. 5-13.

[39] Inamura, T., Geometry of kink microstructure analysed by rank-1 connection, Acta Mater., 2019. 173: p. 270-280. 